\title{
Surgical management for IgG4-related ophthalmic disease by a transcranial biopsy combined with extraorbital decompression: illustrative case
}

\author{
Ryuichi Noda, MD, Tomohiro Inoue, MD, PhD, Sho Tsunoda, MD, and Atsuya Akabane, MD, PhD \\ Department of Neurosurgery, Nippon Telegraph and Telephone Corporation Medical Center Tokyo, Shinagawa-ku, Tokyo, Japan
}

\begin{abstract}
BACKGROUND Immunoglobulin G4-related ophthalmic disease (IgG4-ROD) accounts for $22 \%$ of Japanese lymphoproliferative orbital disease and occurs in $4 \%-34 \%$ of patients with lgG4-related disease, according to the largest case series published to date. Because the optic nerve mass often appears as a tumor-like lesion, it is important, although difficult, to differentiate lgG4-ROD from other orbital tumors and diseases, and biopsy is essential for diagnosis. Here, the authors describe the surgical management of an IgG4-ROD case.

OBSERVATIONS A 63-year-old man presented to the authors' hospital with proptosis and visual impairment. Ophthalmic examination revealed intraocular hypertension. IgG4-related disease with an ophthalmic lesion was suspected on the basis of a blood test and imaging studies. Transcranial biopsy with extraorbital decompression was performed. The patient's symptoms, including visual impairment, improved 3 days after operation, and his IgG4-related disease resolved after corticosteroid treatment.

LESSONS The standard treatment for lgG4-related disease is systemic corticosteroid therapy. However, this treatment should not be administered to patients with IgG4-ROD who a high risk of blindness. In this case, the authors completed a diagnostic and symptom-relieving transcranial biopsy without affecting the patient's aesthetic characteristics. This is the first study, to our knowledge, to report extraorbital decompression via a transcranial approach as a surgical option for lgG4-ROD.
\end{abstract}

https://thejns.org/doi/abs/10.3171/CASE20170

KEYWORDS IgG4-related disease; IgG4-related ophthalmic diseases; transcranial orbital approach; orbital tumor; extraorbital decompression

Immunoglobulin G4-related ophthalmic disease (IgG4-ROD) accounts for $22 \%$ of Japanese lymphoproliferative orbital disease cases ${ }^{1}$ and occurs in $4 \%-34 \%$ of patients with IgG4-related disease, according to the largest case series published to date. ${ }^{2}$ Regarding the effects of $\lg \mathrm{G} 4-\mathrm{ROD}$, lesions in the extraocular muscles or nerves are uncommon, except for lesions in the lacrimal grand..$^{3,4}$

When the optic nerve is affected by $\lg G 4-R O D$, it often appears as a tumor-like lesion, and other tumors have to be excluded for a differential diagnosis. It is important, although difficult, to differentiate lgG4-ROD from other orbital tumors (e.g., lymphoma, optic sheath meningioma, neurofibroma, and schwannoma) and from other similar diseases (e.g., Sjögren syndrome, primary sclerosing cholangitis, Castleman disease, Wegener granulomatosis, and sarcoidosis).5,6 The main difficulty in diagnosis is the poor understanding of the imaging features of lgG4ROD. ${ }^{7}$ The Japanese study group of lgG4-related ophthalmic diseases proposed a diagnostic criterion for IgG4-RODs in 2015, and although the results of blood tests and imaging studies can be strongly suggestive of IgG4-ROD, the definitive diagnosis can be established only on the basis of histopathological results. ${ }^{1}$ Hence, the standard management of lgG4-related disease includes obtaining a biopsy from the most accessible organ and in the least invasive manner and then commencing treatment, typically with corticosteroids.

Our case presentation elaborates on the surgical management of an IgG4-ROD case, which could promptly alleviate symptoms and help to determine the diagnosis at the same time by performing a transcranial extraorbital decompression.

\section{Illustrative Case}

A 63-year-old man was referred to our hospital for further examination of an optic tumor. His chief complaints were right proptosis and vision impairment. He was aware of gradually worsening vision impairment for the past 1.5 years with proptosis development.

ABBREVIATIONS FT = frontotemporal; IgG4-ROD = immunoglobulin G4-related ophthalmic disease; LogMAR = logarithm of the minimum angle of resolution; MRI = magnetic resonance imaging.

INCLUDE WHEN CITING Published February 22, 2021; DOI: 10.3171/CASE20170.

SUBMITTED December 22, 2020. ACCEPTED January 6, 2021.

(C) 2021 The authors, CC BY-NC-ND 4.0 (http://creativecommons.org/licenses/by-nc-nd/4.0/). 
He had recently noticed a relatively rapid aggravation of proptosis and conjunctival injection. Magnetic resonance imaging (MRI) showed a mass in the right orbit. Physical examination revealed proptosis, ptosis, and abduction restriction of the right eye. Ophthalmic examination revealed right visual acuity of 0.7 LogMAR (logarithm of the minimum angle of resolution) in the right eye compared with -0.1 LogMAR in the left eye. The patient's intraocular pressures were $31 \mathrm{~mm} \mathrm{Hg}$ and $15 \mathrm{~mm} \mathrm{Hg}$ in the right and left eyes, respectively.

Contrast-enhanced MRI revealed a contrast-enhanced mass lesion around the right optic nerve sheath (Fig. 1). Contrastenhanced computed tomography of the whole body showed systemic lymphadenopathy that manifested in the right lacrimal gland, bilateral parotid gland, mediastinal lymph nodes, and right inguinal lymph nodes. Blood test results revealed elevated IgG4 concentrations up to $1255 \mathrm{mg} / \mathrm{dL}$. On the basis of the above findings, IgG4ROD was suspected. For a definitive diagnosis and to alleviate symptoms, we performed biopsy via the transcranial approach (Video 1).
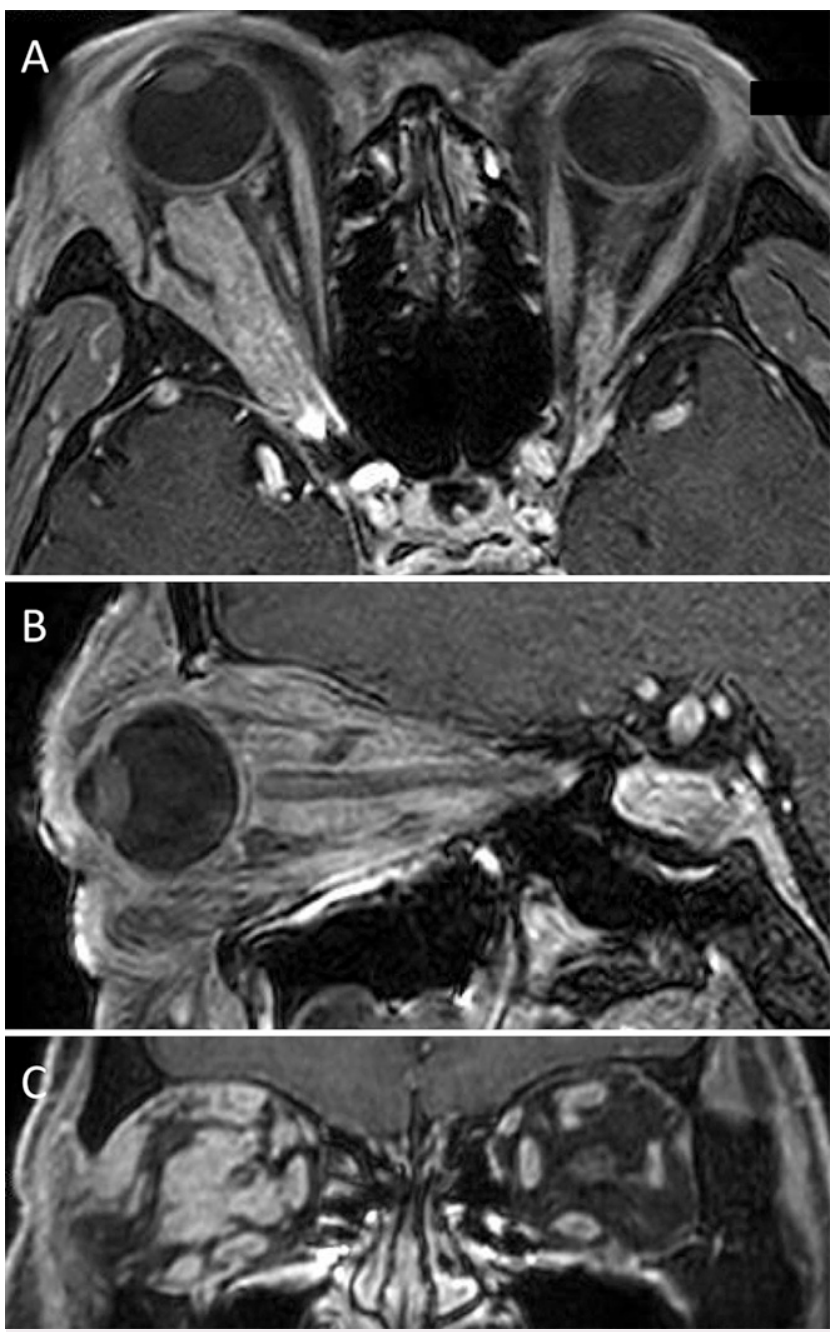

FIG. 1. MRI of the orbit. T1-weighted image with gadolinium contrast of the mass of the orbit and that of the enlarged lacrimal glands. A: Axial view. B: Saggital view. C: Coronal view.
VIDEO 1. The sphenoid ridge drilled off, and the lateral wall of the orbit was flattened. The dura propria was elevated with minimal retraction of the dura, and the meningo-orbital band was incised. After clinoidectomy of the anterior clinoid, the optic canal was drilled out. The lateral wall and the roof of the orbit were drilled out until eggshell thin. The orbital roof and the lateral wall were removed, and the optic sheath was incised. Orbital mass biopsy was performed by using a lateral approach (between superior orbital muscle and lateral orbital muscle) and confirmed the orbital mass as IgG4-ROD. An autogenous temporalis fascia patch graft was used to close the incised orbital sheath in order to maintain decompression. Click here to view.

The surgery was performed with the patient under general anesthesia. Craniotomy was performed using Yaşargil's pterional method. The patient was placed supine. After head fixation in a Mayfield clamp, the head was rotated $30^{\circ}$ contralaterally. The skin incision began at the level of the zygomatic arch at the preauricular region $(1 \mathrm{~cm}$ anterior to the tragus) and curved along the hairline until $1-2 \mathrm{~cm}$ from the midline. The curve was slightly (approximately 1 fingerbreadth) augmented posteriorly at the supra-auricular level to create space for the temporal muscle to retract posteriorly (Fig. 2A). The temporal muscle was incised through the plane between the laminae of the temporal fascia to preserve the frontal branch of the facial nerve. The cutaneous flap was inferiorly reflected. The muscle flap was incised along the linear temporalis, except for the posterior 3-fingerbreadth region, and pushed posteriorly to expose the base of the temporal bone. Craniotomy was designed using 3 burr holes on the cranium (MacCarty's keyhole, 1 hole anterior to the squamous suture at the level of the zygomatic root, and 1 hole inferior to the linear temporalis line; Fig. 2B). The sphenoid ridge was drilled off, and the lateral wall of the orbit was flattened. After elevating the dura propria, with minimal retraction of the dura, we performed clinoidectomy of the anterior clinoid and drilled out the optic canal. The superior and lateral walls of the orbit were sufficiently removed (Figs. $2 \mathrm{C}$ and $3 \mathrm{~A}$ ). The periorbital sheath was incised, and biopsy was performed between the lateral rectus and superior rectus muscles (lateral approach). The orbital sheath was reconstructed with temporal fascia to decrease the intraocular pressure. The histopathological results were consistent with IgG4-related disease. The superior and lateral walls were left open for decompression (Fig. 3B). The bone flap was put back in its original position and fixed to the skull with titanium plates and screws. Calcium phosphate bone paste was used to cover the bone defects. The edges of the temporalis fascia and those of the temporal muscle were sutured to the original position, and the skin was also sutured.

Ophthalmological examination performed 3 days after the operation showed dramatic improvement in visual acuity $(-0.1$ LogMAR in the right eye). Intraocular pressure had decreased to $15 \mathrm{~mm} \mathrm{Hg}$. Postoperative MRI showed significant improvement in proptosis (Fig. 4). After confirming the IgG4-ROD diagnosis on the basis of histopathological results, oral administration of corticosteroids was initiated 7 days postoperatively and continued after discharge. The lesion showed gradual resolution after treatment. During the 6-month follow-up, complete remission was attained, and no recurrence was shown.

\section{Discussion}

\section{Observations}

Although the treatment of IgG4-ROD is hampered by a lack of prospective randomized clinical trials, corticosteroid therapy is 

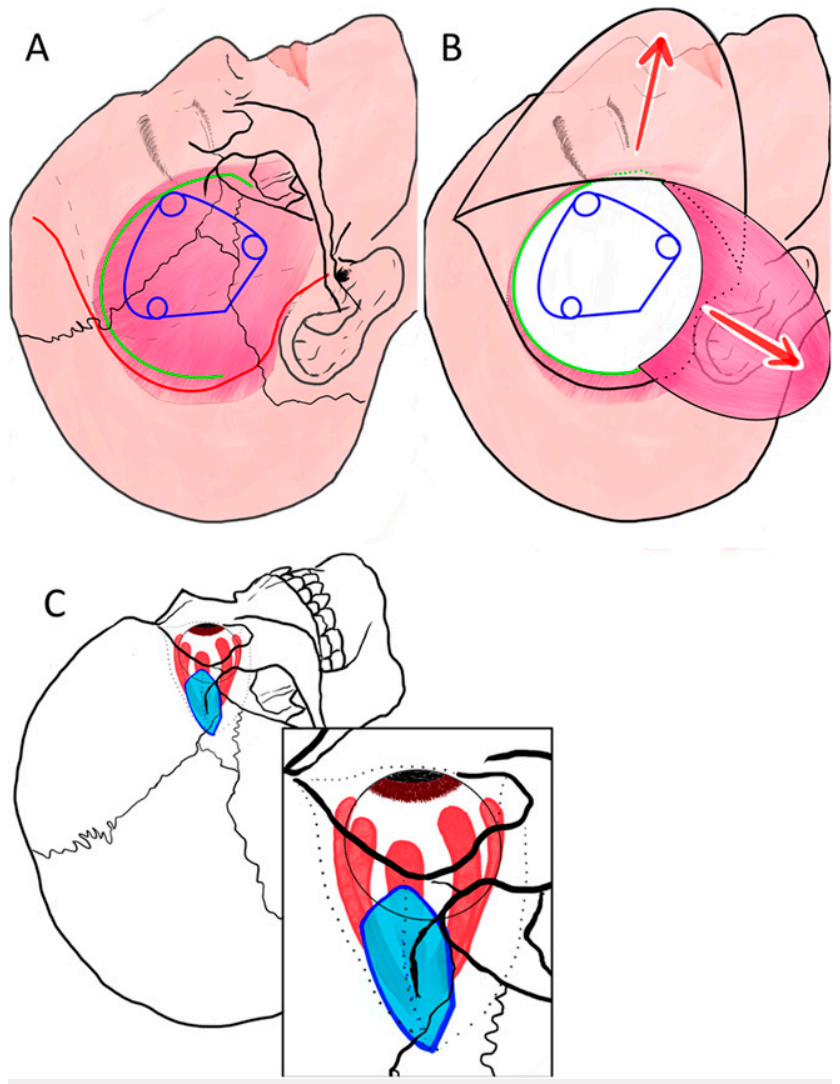

FIG. 2. A: Scheme of skin incision and temporal muscle incision. The skin incision began from the preauricular region at the level of the zygomatic arch. The curve of the incision was slightly (approximately 1 fingerbreadth) augmented posteriorly at the supra-auricular level to create space for the posterior retraction of the temporal muscle, and it was ended at 1-2 cm from the midline (red line). B: Scheme indicating the direction (arrows) of retraction of the skin flap and that of the temporal muscle. The cutaneous flap was reflected inferiorly. The muscle flap was incised along the linear temporalis, except for the posterior 3-fingerbreadth region (green line), and pushed posteriorly to expose the base of the temporal bone. Craniotomy was designed by creating 3 burr holes on the cranium (MacCarty's keyhole, 1 hole posterior to the squamous suture on the level of the zygomatic root, and 1 hole inferior to the linear temporalis line; blue line). C: Scheme of the extraorbital decompression. The lateral and superior orbital walls were drilled off (blue highlighted area).

reported to be a treatment option. , $^{8,9}$ To commence this treatment, a definitive diagnosis must be achieved on the basis of histopathological examination. Thus, biopsy from the most accessible lesion and in the least invasive manner is usually considered. In addition, although corticosteroid treatment is reported to result in a prompt response in IgG4-ROD cases, several weeks are required for remission, ${ }^{7,10}$ and there are concerns about the relatively high recurrence rate $(18 \%-58 \%) .^{11}$ Moreover, in IgG4-ROD with ocular hypertension, corticosteroid treatment could aggravate symptoms by increasing the intraocular pressure. ${ }^{12}$ We could not risk exacerbating the ocular hypertension and causing blindness as a result. Therefore, in cases such as ours, it is important to relieve the optic nerve from intraocular pressure as soon as possible to improve symptoms and prevent permanent nerve damage.
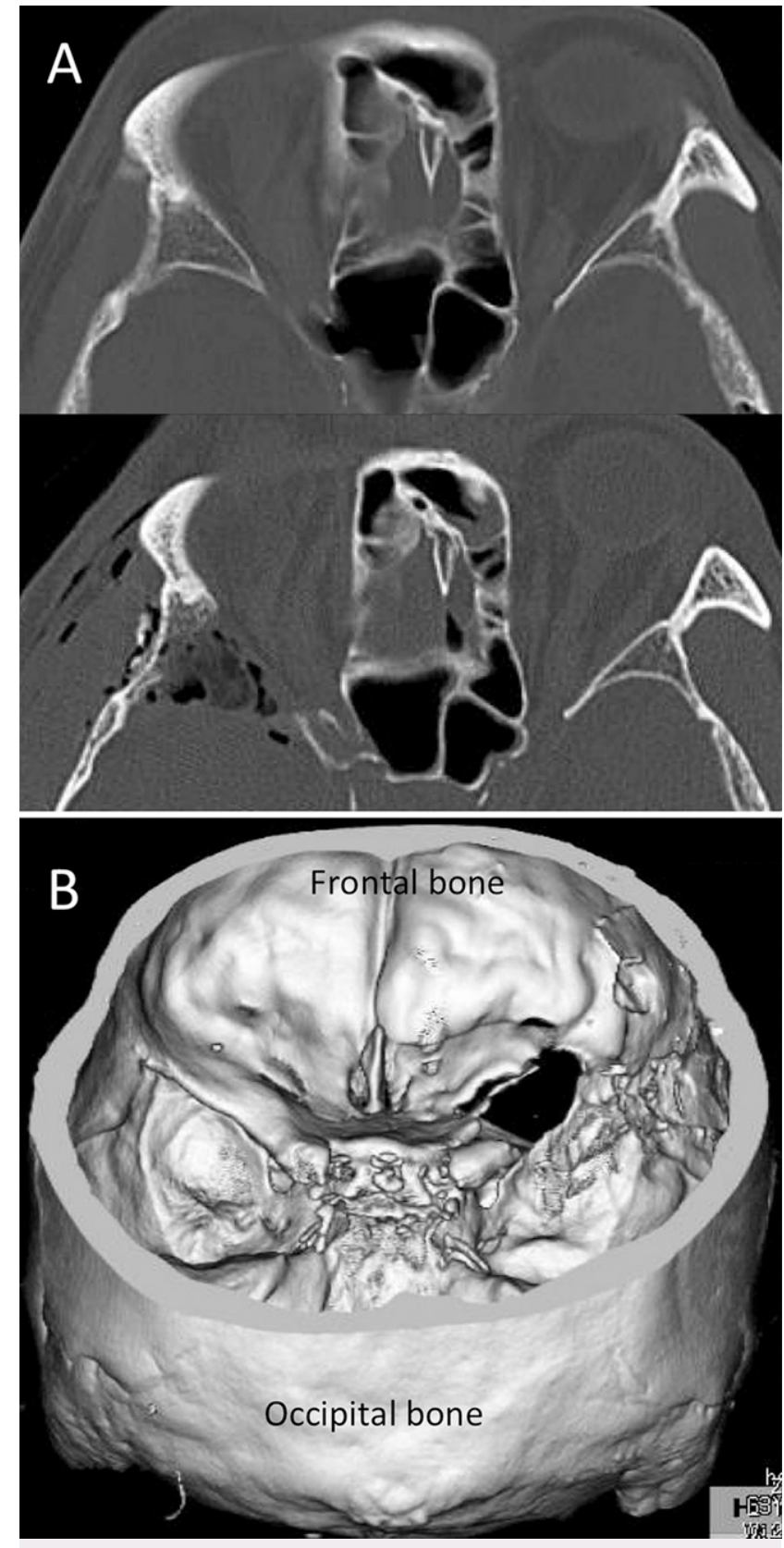

FIG. 3. Postoperative bone computed tomography (CT). Comparison between pre- and postoperative axial CT images of bone (A). Lateral wall of the orbit is drilled off. Posterior view of three-dimensional bone image showing the lateral wall and orbital roof were removed (B).

Ominato et al. ${ }^{13}$ reported a case series of patients with lgG-ROD treated solely with surgical debulking. The results showed that debulking surgery could be a treatment option for lgG4-ROD. However, because of the lack of accumulated investigations and properly designed studies, the efficacy of removing or debulking the tumor remains questionable. ${ }^{14}$ In our case, mass reduction was not necessary. Considering the risk of complications, we decided to avoid debulking surgery. In contrast, an extraorbital decompression was considered effective and feasible. In fact, it has already been reported that medial 


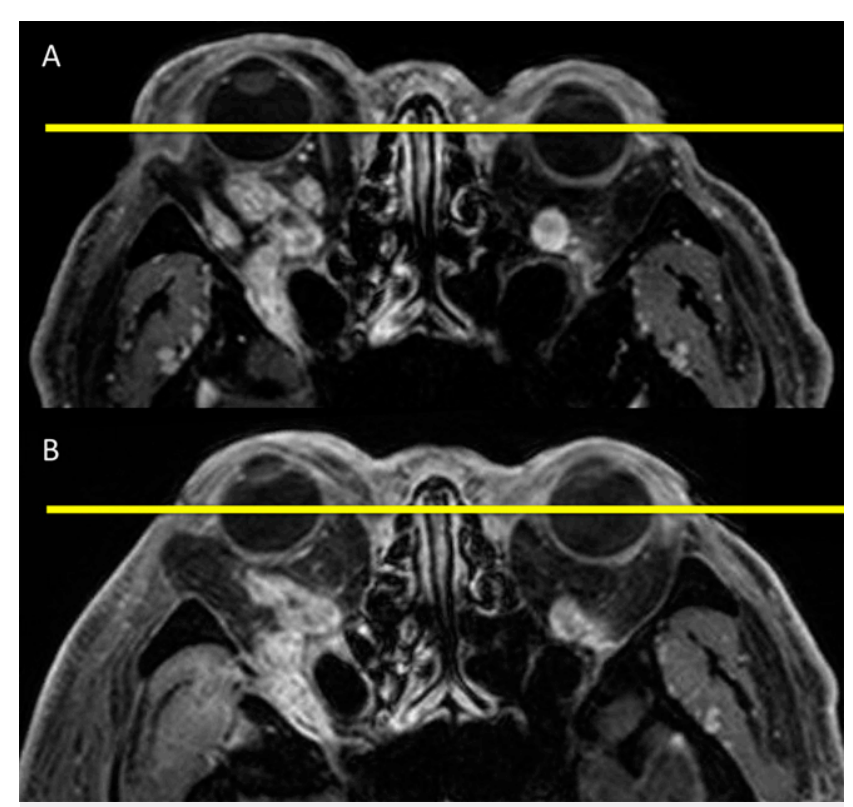

FIG. 4. Proptosis of the right eye (A) dramatically improved, as observed on MRI performed 3 days after operation (B). Equator line of the left eye is shown by the yellow lines.

wall decompression of the orbit is an effective surgical technique to improve exophthalmos caused by thyroid eye disease. ${ }^{15}$ Furthermore, orbital decompression can be an emergency procedure in visionthreatening conditions, such as dysthyroid optic neuropathy, to relieve pressure on the orbital apex and improve vascular/axonal flow within the optic nerve. ${ }^{16}$ We drilled out the optic canal to decrease pressure to the optic nerve, but we did not open its dura to avoid damage to the optic nerve. Transcranial approaches to orbital tumors are already well-established and safe methods. ${ }^{17}$ In the present case, to fulfill our 2 purposes, which were confirming the diagnosis and alleviating the symptoms, biopsy and decompression via the transcranial orbital approach were the best choice. Extracranial decompression of the orbit was a consistent method to decrease intraocular pressure.

The invasiveness, especially in terms of cosmesis, was our concern. The transcranial approach for orbital tumors was initially developed by Dandy in $1941^{18}$ and has been modified ever since. The current concept of the transcranial orbital approach is a frontotemporal (FT) craniotomy with a combination of orbitectomy and zygomectomy. ${ }^{19,20}$ The reconstruction of pterional, temporal, and orbital rim defects after these craniotomies is important to avoid temporal hollowing and maintain functional restoration. Therefore, we left the posterior 3-fingerbreadth region of the temporal muscle unincised, and we completely reconstructed the muscle to prevent atrophy. We also used hydroxyapatite to patch the bone gap. In addition, it is important to achieve optimal cosmesis, especially in cases such as ours, in which we had to perform craniotomy for an extracranial lesion. We fully considered the patient's aesthetic aspect. As a result, in this case, we chose FT craniotomy with preservation of the supraorbital rim and zygoma. Osteotomy of the superior and lateral walls of the orbit was performed via a simple FT craniotomy; orbitectomy and zygomectomy were avoided. The patient had no objective aesthetic deterioration, and the results were also subjectively satisfactory.

\section{Lessons}

In conclusion, we could complete a diagnostic and symptomrelieving transcranial biopsy without affecting the patient's aesthetic characteristics. In patients with IgG4-ROD in critical condition, extraorbital decompression might be an option before administering systemic corticosteroid to prevent blindness. Furthermore, this method could be a possible strategy for any kind of orbital tumors with elevated intraocular pressure.

To the best of our knowledge, this is the first study to report extraorbital decompression and biopsy via the transcranial approach as a surgical technique for lgG4-ROD. This method can be applied in patients with severe visual impairment and elevated ocular pressure.

\section{References}

1. Japanese study group of IgG4-related ophthalmic disease. A prevalence study of lgG4-related ophthalmic disease in Japan. Jpn J Ophthalmol. 2013;57(6):573-579.

2. Ebbo M, Patient M, Grados A, et al. Ophthalmic manifestations in IgG4-related disease: clinical presentation and response to treatment in a French case-series. Medicine (Baltimore). 2017; 96(10):e6205.

3. Masaki Y, Dong L, Kurose N, et al. Proposal for a new clinical entity, IgG4-positive multiorgan lymphoproliferative syndrome: analysis of 64 cases of IgG4-related disorders. Ann Rheum Dis. 2009;68(8):1310-1315.

4. Nagai K, Andoh K, Nakamura N, et al. Suspected idiopathic sclerosing orbital inflammation presenting as immunoglobulin G4-related disease: a case report. J Med Case Rep. 2011;5: 427.

5. Noshiro S, Wanibuchi M, Akiyama Y, et al. IgG4-related disease initially presented as an orbital mass lesion mimicking optic nerve sheath meningioma. Brain Tumor Pathol. 2015;32(4):286-290.

6. Purohit BS, Vargas MI, Ailianou A, et al. Orbital tumours and tumour-like lesions: exploring the armamentarium of multiparametric imaging. Insights Imaging. 2016;7(1):43-68.

7. Yu WK, Tsai CC, Kao SC, et al. Immunoglobulin G4-related ophthalmic disease. Taiwan J Ophthalmol. 2018;8(1):9-14.

8. Hagiya C, Tsuboi H, Yokosawa M, et al. Clinicopathological features of IgG4-related disease complicated with orbital involvement. Mod Rheumatol. 2014;24(3):471-476.

9. Yamamoto M, Yajima H, Takahashi H, et al. Everyday clinical practice in IgG4-related dacryoadenitis and/or sialadenitis: results from the SMART database. Mod Rheumatol. 2015;25(2): 199-204.

10. Hirata Y, Fukae J, Nishida A, et al. Longstanding IgG4-related ophthalmic disease dramatically improved after steroid therapy. Intern Med. 2018;57(19):2879-2883.

11. Suimon $Y$, Kase $S$, Ishijima $K$, et al. A clinicopathological study on IgG4-related ophthalmic disease. Int J Ophthalmol. 2018;11(9): 1539-1544.

12. Kersey JP, Broadway DC. Corticosteroid-induced glaucoma: a review of the literature. Eye (Lond). 2006;20(4):407-416.

13. Ominato J, Oyama $\mathrm{T}$, Cho $\mathrm{H}$, et al. The natural course of $\lg \mathrm{G} 4$ related ophthalmic disease after debulking surgery: a single-centre retrospective study. BMJ Open Ophthalmol. 2019;4(1):e000295.

14. Detiger SE, Karim AF, Verdijk RM, et al. The treatment outcomes in IgG4-related orbital disease: a systematic review of the literature. Acta Ophthalmol. 2019;97(5):451-459.

15. Cheng AMS, Wei YH, Liao SL. Strategies in surgical decompression for thyroid eye disease. Oxid Med Cell Longev. 2020;2020: 3537675. doi:10.1155/2020/3537675

16. Roos JCP, Murthy R. Update on the clinical assessment and management of thyroid eye disease. Curr Opin Ophthalmol. 2019; 30(5):401-406. 
17. Margalit N, Ezer H, Fliss DM, et al. Orbital tumors treated using transcranial approaches: surgical technique and neuroophthalmogical results in 41 patients. Neurosurg Focus. 2007; 23(5):E11.

18. Dandy WE. Orbital tumors: results following the transcranial operative attack. Arch NeurPsych. 1941;46(3):568.

19. Kannan S, Hasegawa M, Yamada Y, et al. Tumors of the orbit: case report and review of surgical corridors and current options. Asian J Neurosurg. 2019;14(3):678-685.

20. Welkoborsky HJ, Plontke SK. Possible surgical approaches to the orbit. Article in German. HNO. 2018;66(11):812-826.

\section{Disclosures}

The authors report no conflict of interest concerning the materials or methods used in this study or the findings specified in this paper.

\section{Author Contributions}

Conception and design: Noda, Inoue. Acquisition of data: Noda. Analysis and interpretation of data: Noda, Inoue. Drafting the article: Noda, Inoue. Critically revising the article: Inoue. Reviewed submitted version of manuscript: Inoue, Tsunoda, Akabane. Administrative/technical/ material support: Akabane. Study supervision: Akabane.

\section{Supplemental Information}

Videos

Video 1. https://vimeo.com/498368462.

\section{Correspondence}

Ryuichi Noda: Nippon Telegraph and Telephone Corporation Medical Center Tokyo, Shinagawa-ku, Tokyo, Japan. rnrn46_8447@yahoo.co.jp. 